\title{
Effects of chronic scopolamine treatment on cognitive impairment and neurofilament expression in the mouse hippocampus
}

\author{
JAE-CHUL LEE $^{1 *}$, JOON HA PARK $^{2 *}$, JI HYEON AHN $^{2}$, JINSEU PARK $^{2}$, IN HYE KIM $^{1}$, JEONG HWI CHO $^{1}$, \\ BICH NA SHIN ${ }^{1}$, TAE-KYEONG LEE ${ }^{1}$, HYUNJUNG KIM ${ }^{1}$, MINAH SONG ${ }^{1}$, GEUM-SIL CHO ${ }^{3}$, DAE WON KIM ${ }^{4,5}$, \\ IL JUN KANG $^{6}$, YOUNG-MYEONG KIM ${ }^{7}$, MOO-HO WON ${ }^{1}$ and SOO YOUNG CHOI ${ }^{2}$ \\ ${ }^{1}$ Department of Neurobiology, School of Medicine, Kangwon National University, Chuncheon, Gangwon 24341; \\ ${ }^{2}$ Department of Biomedical Science, Research Institute of Bioscience and Biotechnology, Hallym University, \\ Chuncheon, Gangwon 24252; ${ }^{3}$ Pharmacology and Toxicology Department, Shinpoong Pharmaceutical Co., Ltd., \\ Ansan, Gyeonggi 15610; ${ }^{4}$ Department of Biochemistry and Molecular Biology; ${ }^{5}$ Research Institute of Oral Sciences, \\ College of Dentistry, Kangnung-Wonju National University, Gangneung, Gangwon 25457; ${ }^{6}$ Department of Food \\ Science and Nutrition, Hallym University, Chuncheon, Gangwon 24252; ${ }^{7}$ Department of Molecular and Cellular \\ Biochemistry, School of Medicine, Kangwon National University, Chuncheon, Gangwon 24341, Republic of Korea
}

Received August 17, 2017; Accepted November 9, 2017

DOI: $10.3892 / \mathrm{mmr} .2017 .8082$

\begin{abstract}
Neurofilaments (NFs) including neurofilament-200 kDa (NF-H), neurofilament-165 kDa (NF-M) and neurofilament- $68 \mathrm{kDa}(\mathrm{NF}-\mathrm{L})$ are major protein constituents of the brain, and serve important roles in the regulation of axonal transport. NF alteration is a key feature in the pathogenesis of neurological disorders involving cognitive dysfunction. In the present study, cognitive impairments were investigated, via assessments using the Morris water maze and passive avoidance tests, in mice following chronic systemic treatment with $1 \mathrm{mg} / \mathrm{kg}$ scopolamine (SCO) for 4 weeks. SCO-induced cognitive impairments were significantly observed 1 week following the SCO treatment, and these cognitive deficits were maintained for 4 weeks. However, the NF immunoreactivities and levels were altered differently according to the hippocampal subregion following SCO treatment. NF-H immunoreactivity and levels were markedly altered in all hippocampal subregions, and were significantly increased 1 week following the
\end{abstract}

Correspondence to: Professor Moo-Ho Won, Department of Neurobiology, School of Medicine, Kangwon National University, 1 Kangwondaehak, Chuncheon, Gangwon 24341, Republic of Korea E-mail: mhwon@kangwon.ac.kr

Professor Soo Young Choi, Department of Biomedical Science, Research Institute of Bioscience and Biotechnology, Hallym University, 1 Hallymdaehak, Chuncheon, Gangwon 24252, Republic of Korea

E-mail: sychoi@hallym.ac.kr

${ }^{*}$ Contributed equally

Key words: scopolamine, cognitive deficit, hippocampus proper, dentate gyrus, neurofilaments
SCO treatment; thereafter, the immunoreactivity and levels significantly decreased with time. NF-M immunoreactivity and levels gradually decreased in the hippocampus and were significantly decreased 4 weeks following SCO treatment. NF-L immunoreactivity and levels gradually decreased in the hippocampus, and were significantly decreased 2 and 4 weeks following SCO treatment. In conclusion, the results of the present study demonstrated that chronic systemic treatment with SCO induced cognitive impairment from 1 week following SCO treatment, and NF expression was diversely altered according to the hippocampal subregion from 1 week following SCO treatment. These results suggest that SCO-induced changes in NF expression may be associated with cognitive impairment.

\section{Introduction}

Scopolamine (SCO), a non-selective muscarinic acetylcholine receptor antagonist, is known to interfere with cholinergic transmission in the brain $(1,2)$. Recently, we have reported that the SCO-induced dysregulation of cholinergic activity in the hippocampus leads to interference in processes of learning and memory in mice (3-5). Thus, SCO has been used as a pharmacological tool for the development of experimental animal models of cognitive deficits; many researchers have identified the models as a candidate for the treatment of Alzheimer's disease (6-8).

The hippocampus or hippocampal formation, which consists of the hippocampus proper (CA1-3 areas) and dentate gyrus, displays critical roles in cognitive processes such as learning, memory consolidation, and information retrieval (9-12). Memory loss followed by cognitive decline is found to be a common feature of various neurological disorders like Alzheimer's disease (13). The cholinergic system has long been known to be critically involved in hippocampal-dependent cognitive function $(14,15)$. Deficits in the hippocampal 
cholinergic system can affect learning and memory; namely, the loss of cholinergic function in the hippocampus is correlated with significant cognitive impairments $(16,17)$.

The cytoskeleton extends throughout the cytoplasm of cell and provides a number of important functions, such as the maintenance of cellular shape, holding cellular organelles in place, and assisting in the transportation of communication signals between cells (18). Abnormalities of the neuronal cytoskeleton frequently represent a key feature of Alzheimer's disease, in which the disruption of the normal cytoskeletal framework of neurons typically triggers dystrophic neurites (19).

Neurofilaments (NFs) do not only support the morphology of neurons as cytoskeletal components but also play a fundamental role in axonal transport (18). NFs are composed of three major subunits, neurofilament-200 kDa (NF-H), neurofilament-165 kDa (NF-M) and neurofilament-68 kDa (NF-L); according to observed molecular weight (20). NF-H and NF-M are involved in the stabilization of newly-sprouted axonal processes $(21,22)$, and NF-L is expressed in axonal growth cones (23). In particular, NF-H plays an important role in the stabilization and maturation of pre-existing connections (24). Previous studies have suggested that NFs are closely related to neurodegenerative diseases (25-28). The pathological disruption of axonal NFs results in the breakdown and fasciculation of the filaments, and its most notable characteristic is whorls of NFs and ring-like structures in swollen axons $(28,29)$.

However, few studies regarding changes in NFs expressions in animal models of SCO-induced amnesia have been reported. Therefore, in this study, we examined chronological changes in NFs immunoreactivities and levels of NFs proteins in the mouse hippocampus, as well as in memory impairments following chronic systemic treatment with SCO.

\section{Materials and methods}

Experimental animals and SCO treatment. Male ICR mice (B.W., 25-30 g; 8 weeks of age) were purchased from the Orient Bio Inc. (Seongnam, South Korea). The procedures for animal handling and care, and experimental protocols were reviewed and approved based on ethical procedures and scientific care by the Kangwon National University-Institutional Animal Care and Use Committee (KW-130424-2).

Mice were intraperitoneally injected with $1 \mathrm{mg} / \mathrm{kg}$ of SCO (Sigma-Aldrich; Merck KGaA, Darmstadt, Germany), once daily, for 1,2, 3 and 4 weeks ( $n=14$ at each point in time). Dose of SCO was selected based on previous studies $(30,31)$. The control mice ( $n=14$ at each point in time) were injected with the same volume of saline ( $\mathrm{pH}$ 7.4). The mice were sacrificed 1, 2, 3 and 4 weeks after SCO or saline treatment.

Passive avoidance test. Short-term memory ability was evaluated by assessing the latency of passive avoidance test. Animals $(n=7)$ in each group were tested 1 day before sacrifice following training for 1 day. In short, according to our published procedure (32), the test was performed with an apparatus that consisted of two compartments (light and dark) with a grid floor (GEM 392; San Diego Instruments., San Diego, CA, USA). In training session, mice were allowed to explore environments in both light and dark compartments for $1 \mathrm{~min}$ and given an inescapable foot-shock $(0.3 \mathrm{~mA}$ for
$3 \mathrm{sec}$ ) after entering the dark compartment. Test session was performed 15 min after the training session using the same paradigm, but without applying the foot-shock. The interval between the starting of the test session and mouse's entry into the dark compartment was defined as the latency time of passive avoidance test. When mouse did not enter the dark room within $180 \mathrm{sec}$, the latency was recorded as $180 \mathrm{sec}$.

Water maze performance. The animals used in the passive avoidance test were used for spatial learning and memory test using the Morris water maze task according to our published protocol (4). In short, a circular pool $(90 \mathrm{~cm}$ in diameter and $45 \mathrm{~cm}$ height) filled with water was conceptually divided into four quadrants, and a platform $(6 \mathrm{~cm}$ in diameter and $29 \mathrm{~cm}$ in height) was placed in one quadrant $1 \mathrm{~cm}$ below the water surface. Test was conducted on the last day of every week after saline or SCO treatment, and training was conducted before the test for 3 consecutive days (saline and SCO were administered continuously during training and test periods). Mice ( $n=7$ in each group) were allowed to swim for $120 \mathrm{sec}$ to search for the hidden platform. If they failed to locate the platform within $120 \mathrm{sec}$, escape would be assisted and escape latency was recorded as $120 \mathrm{sec}$. At the end of each trial, each mouse would stay on the platform for $3 \mathrm{sec}$. Escape latency (the time taken to find the platform) was recorded with Noldus Ethovision video tracking system (EthoVision XT; Noldus Information Technology, Wageningen, The Netherlands).

Western blot analysis. NFs levels in the hippocampal formation ( $n=7$ at each point in time) were analyzed according to our published procedure (32). In brief, hippocampal tissues were homogenized and the protein level was determined using a Micro bicinchoninic acid (BCA) protein assay kit (Pierce Biotechnology, Inc., Rockford, IL, USA). The membranes were incubated with rabbit anti- NF-H, NF-M and NF-L (1:1,000; Chemicon International, Inc., Billerica, MA, USA), and exposed to peroxidase conjugated goat anti-rabbit IgG (Santa Cruz Biotechnology, Inc., Dallas, TX, USA) and an enhanced luminol-based chemiluminescent (ECL) kit (Pierce Biotechnology, Inc.).

The result of the western blot analysis was scanned, and densitometric analysis for the quantification of the bands was done using Scion Image software (Scion Corp., Frederick, MD, USA), which was used to count ROD. A ratio of the ROD was calibrated as $\%$, with control group designated as $100 \%$.

Immunohistochemistry. According to our published procedure (33), in brief, mice ( $n=7$ at each point in time) were anesthetized with pentobarbital sodium $(40 \mathrm{mg} / \mathrm{kg}$, JW Pharmaceutical Co., Ltd., Seoul, Korea) and perfused with $4 \%$ paraformaldehyde. Brain tissues were serially sectioned in a cryostat (Leica, Wetzlar, Germany) into 30- $\mu$ m coronal sections. The sections were incubated with diluted rabbit anti-NF-H (1:1,000; Chemicon International, Inc., Temecula, CA, USA), NF-M (1:1,000; Chemicon International, Inc.) or NF-L (1:1,000; Chemicon International, Inc.), and exposed to biotinylated goat anti-rabbit IgG and streptavidin peroxidase complex (Vector Laboratories, Inc., Burlingame, CA, USA). Finally, they were visualized with 3,3'-diaminobenzidine tetrachloride (Sigma-Aldrich; Merck KGaA). 
Statistical analysis. All data are presented as mean \pm SEM. The normality test was performed using Kolmogorov and Smirnov test for testing normal distributions, and Bartlett test for testing identical standard distributions. All data passed normality test. A multiple-sample comparison was applied to test the differences between groups (ANOVA and the Tukey multiple range test as post hoc test using the criterion of the least significant differences). Statistical analysis was performed using GraphPad Instat (Instat Statistics; GraphPad Software, Inc., La Jolla, CA, USA). P $<0.05$ was considered to indicate a statistically significant difference.

\section{Results}

Avoidance memory defect. To investigate the SCO-induced short-term memory impairment, exploratory preference was measured by passive avoidance test which was reflected by decrease of the latency to enter the dark compartment (Fig. 1). There was no significant difference in the latency time until 4 weeks in the vehicle-treated mice (control mice). However, the latency time of the SCO-treated mice was significantly reduced 1 week after SCO treatment compared to that in the vehicle-treated mice, and the latency time was maintained until 4 weeks after SCO treatment.

Spatial memory defect. Hippocampal dependent spatial learning and memory defect induced by SCO was evaluated using the Morris water maze test. As shown in Table I, the vehicle-treated mice readily learned and memorized the location of the submerged hidden platform. However, in the SCO-treated mice, the escape latency 1 week after SCO treatment was significantly longer than that in the vehicle-treated mice, and the increased escape latency was maintained until 4 weeks after SCO treatment.

Changes in levels of NFs proteins. In the western blot study, we found that change patterns in levels of NF-H, NF-M and NF-L proteins in the hippocampus after SCO treatment were generally different according kinds of the proteins (Fig. 2). One week after SCO treatment, only NF-H level was significantly increased compared with that of the control group. Two weeks after SCO treatment, only NF-L level was significantly decreased compared with that of the control group. Four weeks after SCO treatment, all the NFs levels were significantly decreased compared with those of the control group.

In our preliminary study, we found that there were no significant differences in protein levels of NFs in the hippocampus between 3 and 4 weeks SCO-treated groups. For reference, we recently reported that significant difference in the protein level of myelin basic protein, which is most abundant structural protein in the myelin, was not found in the hippocampus between 3 and 4 weeks SCO-treated groups (4).

Change in NF-H immunoreactivity. CA1 region: In the control group, NF-H immunoreactivity was shown in the stratum pyramidale, and in cells of stratum oriens and radiatum, which had NF-H immunoreactive processes (Fig. 3Aa and Ab). In the SCO-treated mice, NF-H-immunoreactive cells were increased in the stratum oriens and radiatum 1 week after SCO treatment, although the immunoreactivity in the stratum

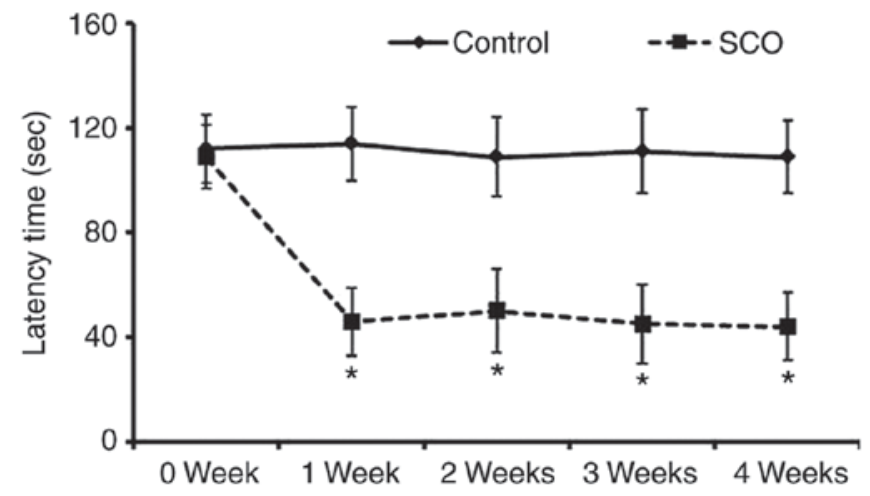

Figure 1. Effect of chronic SCO treatment on the passive avoidance test. Data are presented as the mean \pm standard error of the mean ( $n=7 /$ group). ${ }^{*} \mathrm{P}<0.05$ vs. control. SCO, scopolamine.

pyramidale was not significantly changed compared with that in the control mice (Fig. 3Bb). Two and 4 weeks after $\mathrm{SCO}$ treatment, NF-H immunoreactive cells in the stratum oriens and radiatum were not detected, although the immunoreactivity in the stratum pyramidale was not significantly changed compared with that in the control (Fig. $3 \mathrm{Cb}$ and $\mathrm{Db}$ ).

CA2/3 region: In the control group, the distribution pattern of NF-H immunoreactivity in the CA2/3 region was similar to that in the CA1 region of the control (Fig. 3Aa and Ac). In the SCO-treated mice, NF-H immunoreactive cells and NF-H immunoreactivity were significantly increased 1 week after SCO treatment to the control (Fig. 3Bc). Two and 4 weeks after SCO treatment, NF-H-immunoreactive cells were rarely detected in the stratum oriens and radiatum, and, 4 weeks after SCO treatment, NF-H immunoreactivity in the stratum pyramidale was more significantly decreased compared with that in the 2 weeks SCO-treated group (Fig. 3Cc and Dc).

Dentate gyrus: In the control group, NF-H immunoreactivity was mainly found in the granular cell layer and many cells of the polymorphic layer (Fig. 3Aa and Ad). In the SCO-treated mice, NF-H immunoreactivity in the granular cell layer and many cells of the polymorphic layer was gradually decreased with time after SCO treatment (Fig. 3Bd-Dd).

In the preliminary study, we did not find any difference in NF-H immunoreactivity in the hippocampus between 3 and 4 weeks SCO-treated groups.

Change in NF-M immunoreactivity. CA1 region: Strong NF-M immunoreactivity was observed in fibers in the stratum oriens in the control mice, and NF-M-immunoreactive fibers were scattered in the other layers (Fig. 4Aa and $\mathrm{Ab}$ ). In the SCO-treated mice, NF-M immunoreactivity in the CA1 region was gradually reduced after SCO treatment and significantly decreased 4 weeks after $\mathrm{SCO}$ treatment (Fig. 4Bb-Db).

CA2/3 regions: In the control mice, NF-M immunoreactivity was strongly detected in all the layers (Fig. 4Aa and Ac). In the SCO-treated mice, the distribution pattern of NF-M immunoreactivity was not significantly changed in all the layers until 4 weeks after SCO treatment (Fig. 4Bc-Dc).

Dentate gyrus: In the control mice, NF-M immunoreactivity was mainly detected in the polymorphic layer (Fig. 4Aa and Ad). In the SCO-treated mice, NF-M immunoreactivity was slightly decreased 1 and 2 weeks after SCO 
Table I. Effect of chronic scopolamine treatment on the Morris water maze test.

\begin{tabular}{|c|c|c|c|c|}
\hline \multirow[b]{2}{*}{ Group } & \multicolumn{2}{|c|}{ Training escape latency (sec) } & \multicolumn{2}{|c|}{ Test escape latency (sec) } \\
\hline & Day 1 & Day 2 & Day 3 & Day 4 \\
\hline \multicolumn{5}{|l|}{ Control } \\
\hline 0 weeks & $68.2 \pm 5.5$ & $60.4 \pm 4.2$ & $49.8 \pm 5.8$ & $43.6 \pm 7.9$ \\
\hline 1 week & $66.3 \pm 3.9$ & $59.2 \pm 3.2$ & $46.3 \pm 4.2$ & $41.2 \pm 6.2$ \\
\hline 2 weeks & $69.8 \pm 4.7$ & $61.6 \pm 3.9$ & $45.2 \pm 5.5$ & $39.8 \pm 7.1$ \\
\hline 3 weeks & $64.1 \pm 4.6$ & $57.7 \pm 8.1$ & $42.6 \pm 3.1$ & $35.5 \pm 8.0$ \\
\hline 4 weeks & $67.5 \pm 5.2$ & $54.4 \pm 3.9$ & $43.5 \pm 6.2$ & $33.4 \pm 9.1$ \\
\hline \multicolumn{5}{|l|}{$\mathrm{SCO}$} \\
\hline 0 weeks & $65.7 \pm 3.5$ & $55.7 \pm 4.1$ & $44.6 \pm 5.5$ & $40.1 \pm 8.2$ \\
\hline 1 week & $77.4 \pm 5.7^{\mathrm{a}}$ & $78.5 \pm 7.3^{\mathrm{a}}$ & $76.5 \pm 8.2^{\mathrm{a}}$ & $79.3 \pm 9.0^{\mathrm{a}}$ \\
\hline 2 weeks & $73.5 \pm 6.3^{\mathrm{a}}$ & $75.2 \pm 5.9^{\mathrm{a}}$ & $74.9 \pm 7.8^{\mathrm{a}}$ & $75.7 \pm 10.3^{\mathrm{a}}$ \\
\hline 3 weeks & $79.6 \pm 7.2^{\mathrm{a}}$ & $77.4 \pm 6.2^{\mathrm{a}}$ & $75.7 \pm 6.8^{\mathrm{a}}$ & $77.9 \pm 7.4^{\mathrm{a}}$ \\
\hline 4 weeks & $80.2 \pm 9.3^{\mathrm{a}}$ & $81.8 \pm 6.6^{\mathrm{a}}$ & $77.4 \pm 5.7^{\mathrm{a}}$ & $75.8 \pm 9.2^{\mathrm{a}}$ \\
\hline
\end{tabular}

Escape latency time in seconds in the control and SCO-treated groups during training and test periods ( $\mathrm{n}=7 /$ group). ${ }^{\mathrm{a}} \mathrm{P}<0.05 \mathrm{vs}$. control group. SCO, scopolamine.

treatment and significantly reduced 4 weeks after SCO treatment compared with that in the control (Fig. 4Bd-Dd).

In the preliminary study, there was no significant difference in NF-M immunoreactivity in the hippocampus between 3 and 4 weeks SCO-treated groups.

Change in NF-L immunoreactivity. CA1 region: NF-L immunoreactivity in the control group was weakly detected in all the layers (Fig. 5Aa and Ab). In the SCO-treated group, NF-L immunoreactivity was not significantly changed at any time after SCO treatment (Fig. 5Bb-Db).

CA2/3 region: In the control mice, NF-L immunoreactivity in the $\mathrm{CA} 2 / 3$ region was stronger than that in the $\mathrm{CA} 1$ region (Fig. 5Aa and Ac). In the SCO-treated mice, NF-L immunoreactivity was significantly increased in the stratum pyramidale 1 week after SCO treatment compared with that in the control (Fig. 5Bc), thereafter, NF-L immunoreactivity was gradually decreased in all the layers and very low 4 weeks after SCO treatment (Fig. 5Cc and Dc).

Dentate gyrus: In the control mice, strong NF-L immunoreactivity was found in the polymorphic layer (Fig. 5Aa and Ad). In the SCO-treated mice, NF-L immunoreactivity was not changed 1 week after SCO treatment compared to that in the control (Fig. 5Bd). However, NF-L immunoreactivity significantly reduced 2 weeks after SCO treatment and more significantly decreased 4 weeks after SCO treatment (Fig. 5Cd and Dd).

In our preliminary study, no significant difference in NF-L immunoreactivity was observed in the hippocampus between 3 and 4 weeks SCO-treated groups.

\section{Discussion}

Memory loss followed by cognitive decline is found to be a common feature of various neurological disorders, which may be due to an impairment in the neuronal circuit activity
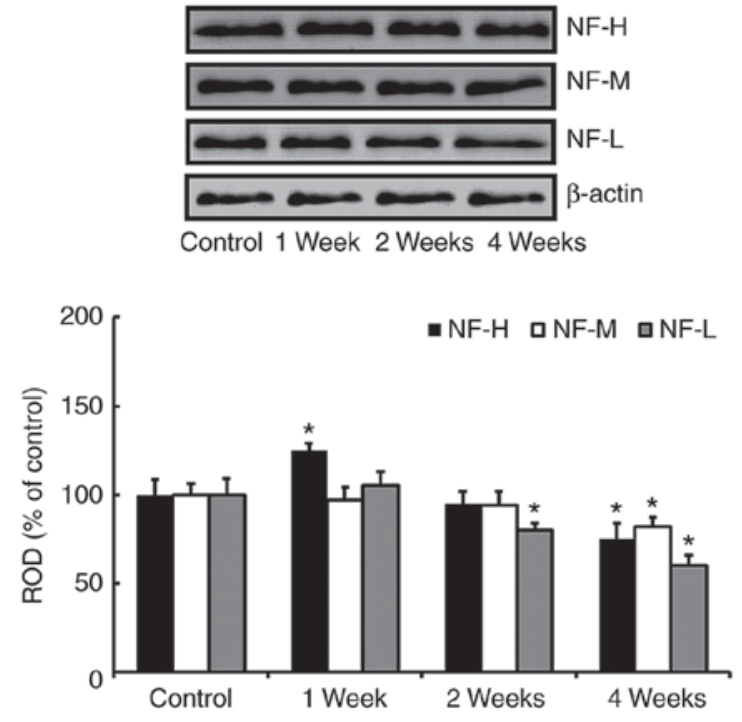

Figure 2. Western blot analysis of NF-H, NF-M and NF-L in the hippocampus of the control and SCO-treated groups. ROD as percentages of the immunoblotting bands are also presented Data are presented as the mean \pm standard error of the mean ( $n=7 /$ group). ${ }^{*} \mathrm{P}<0.05$ vs. control. NF-H, neurofilament-200 kDa; NF-M, neurofilament-165 kDa; NF-L, neurofilament-68 kDa; SCO, scopolamine; ROD, relative optical density.

in the hippocampus (13). Rodent models of cognitive deficits have been established using SCO-induced amnesia (34). In particular, SCO-induced cognitive impairment in animals is similar to that observed in the dysfunction of $\mathrm{AD}(6,7,35)$. Therefore, the SCO-induced memory impairment model has served as a useful tool with which to investigate learning and memory processes.

SCO-induced cognitive deficits have been demonstrated using various behavioral tasks $(3,36)$. In the present study, the SCO-induced cognitive impairment was assessed by the passive avoidance and Morris water maze tests. The passive 

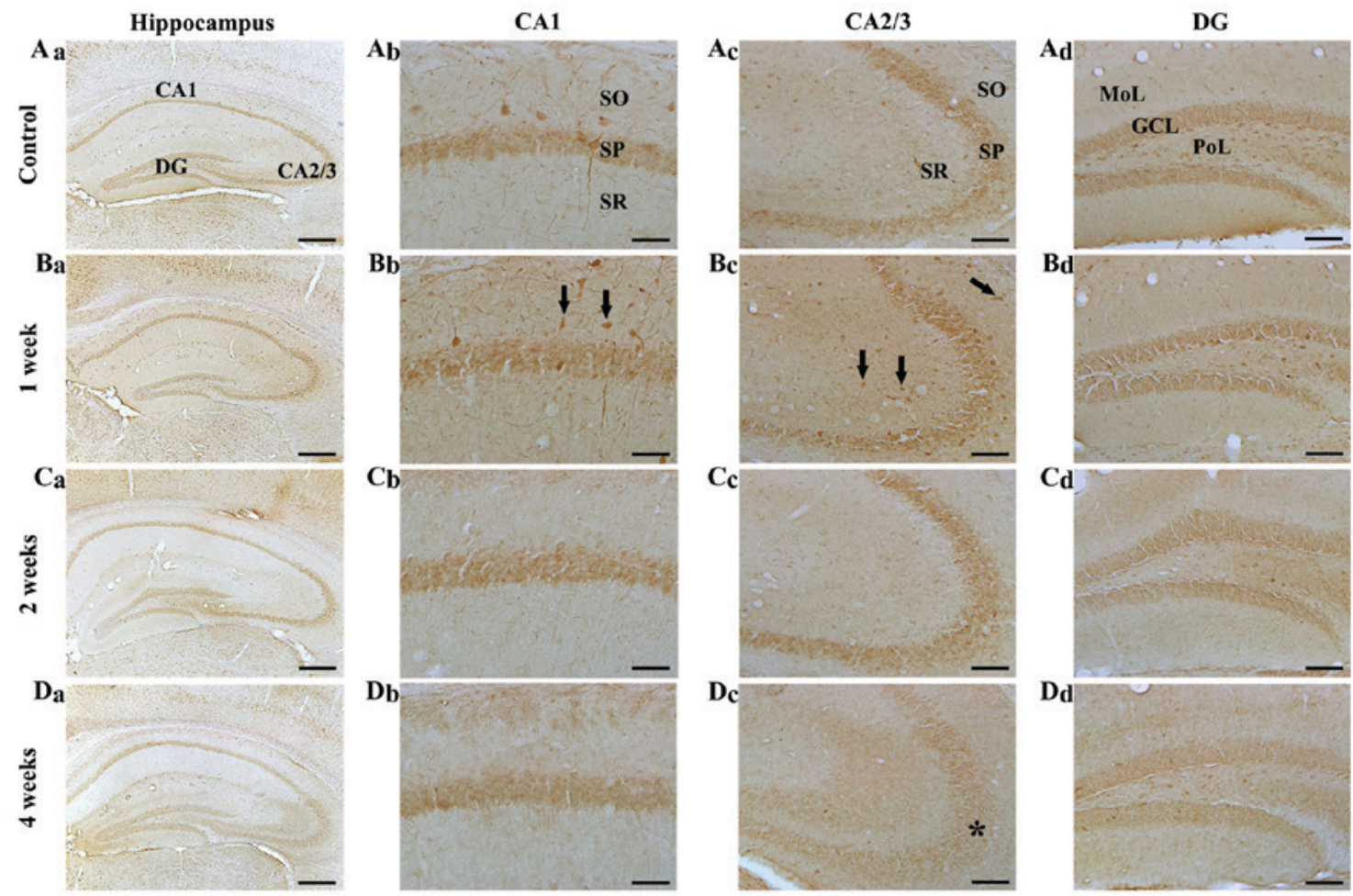

Figure 3. NF-H immunohistochemistry in the hippocampal subregions of the (A) control and SCO-treated mice following (B) 1 , (C) 2 and (D) 4 weeks. NF-H immunoreactive cells (indicated by arrows) increased in the CA1-3 regions ( $\mathrm{Bb}$ and $\mathrm{Bc}$ ) 1 week following scopolamine treatment. A total of 2 and 4 weeks following SCO treatment (C and D, a-d), NF-H immunoreactivity decreased in all hippocampal subregions, in particular, in the stratum pyramidale (indicated by SP and an asterisk) of the (Dc) CA2/3 region 4 weeks following SCO treatment. Scale bars $=800 \mu \mathrm{m}(\mathrm{Aa}-\mathrm{Da}), 50 \mu \mathrm{m}(\mathrm{Ab}-\mathrm{Db})$ and $100 \mu \mathrm{m}$ (Ac-Dc and Ad-Dd). CA, cornus ammonis; DG, dentate gyrus; GCL, granule cell layer; Mol, molecular layer; PoL, polymorphic layer; SO, stratum oriens; SR, stratum radiatum; SP, stratum pyramidale; NF-H, neurofilament-200 kDa; SCO, scopolamine.
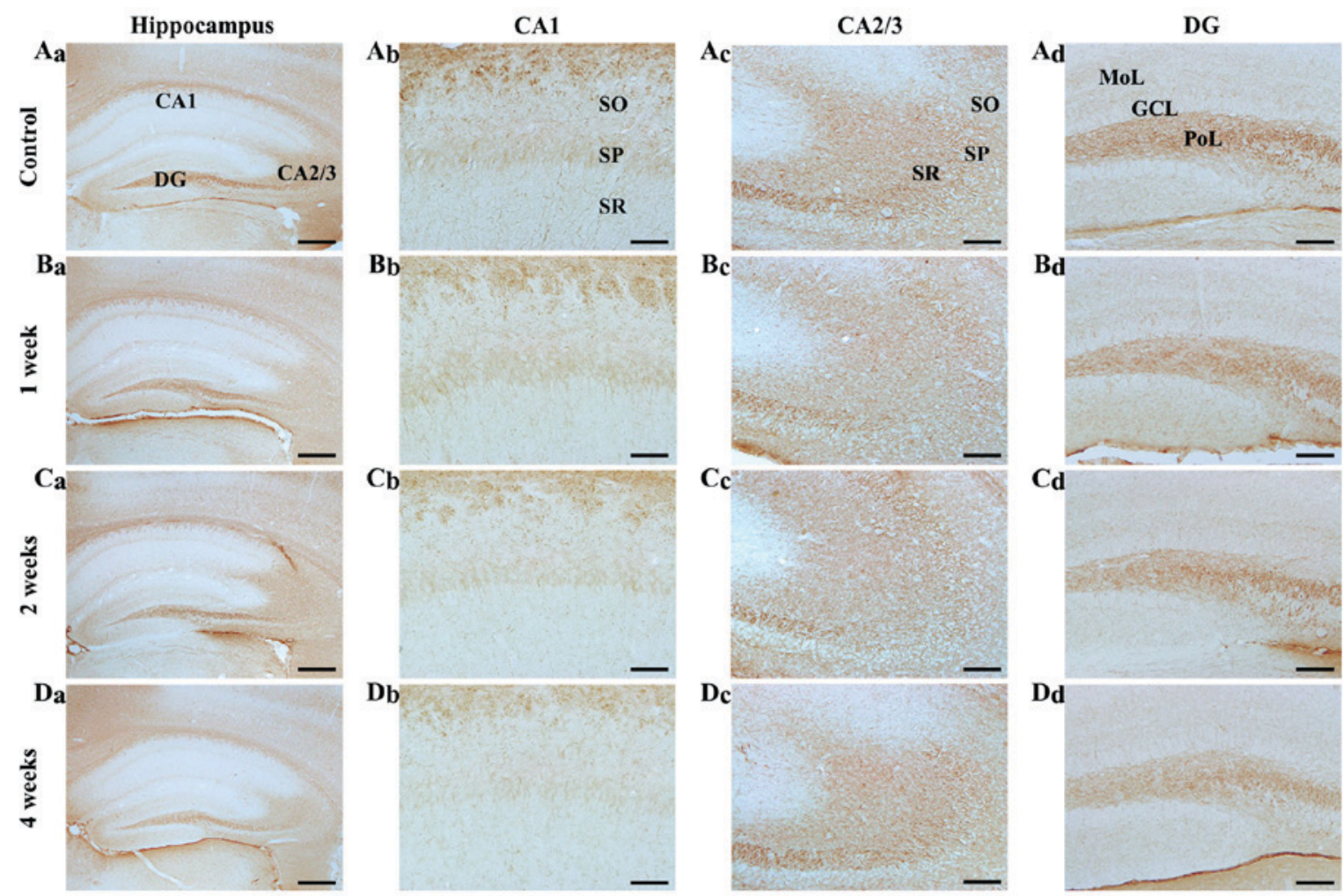

Figure 4. NF-M immunohistochemistry in the hippocampal subregions of the (A) control and SCO-treated mice following (B) 1, (C) 2 and (D) 4 weeks. NF-M immunoreactivity in all hippocampal subregions of the SCO-treated mice were not markedly altered until 2 weeks following SCO treatment; however, NF-M immunoreactivity was decreased (Da-d) 4 weeks following SCO treatment. Scale bar=800 $\mu \mathrm{m}$ (Aa-Da), $50 \mu \mathrm{m}$ (Ab-Db) and $100 \mu \mathrm{m}$ (Ac-Dc and Ad-Dd). CA, cornus ammonis; DG, dentate gyrus; GCL, granule cell layer; Mol, molecular layer; PoL, polymorphic layer; SO, stratum oriens; SP, stratum pyramidale; SR, stratum radiatum; NF-M, neurofilament-165 kDa; SCO, scopolamine. 

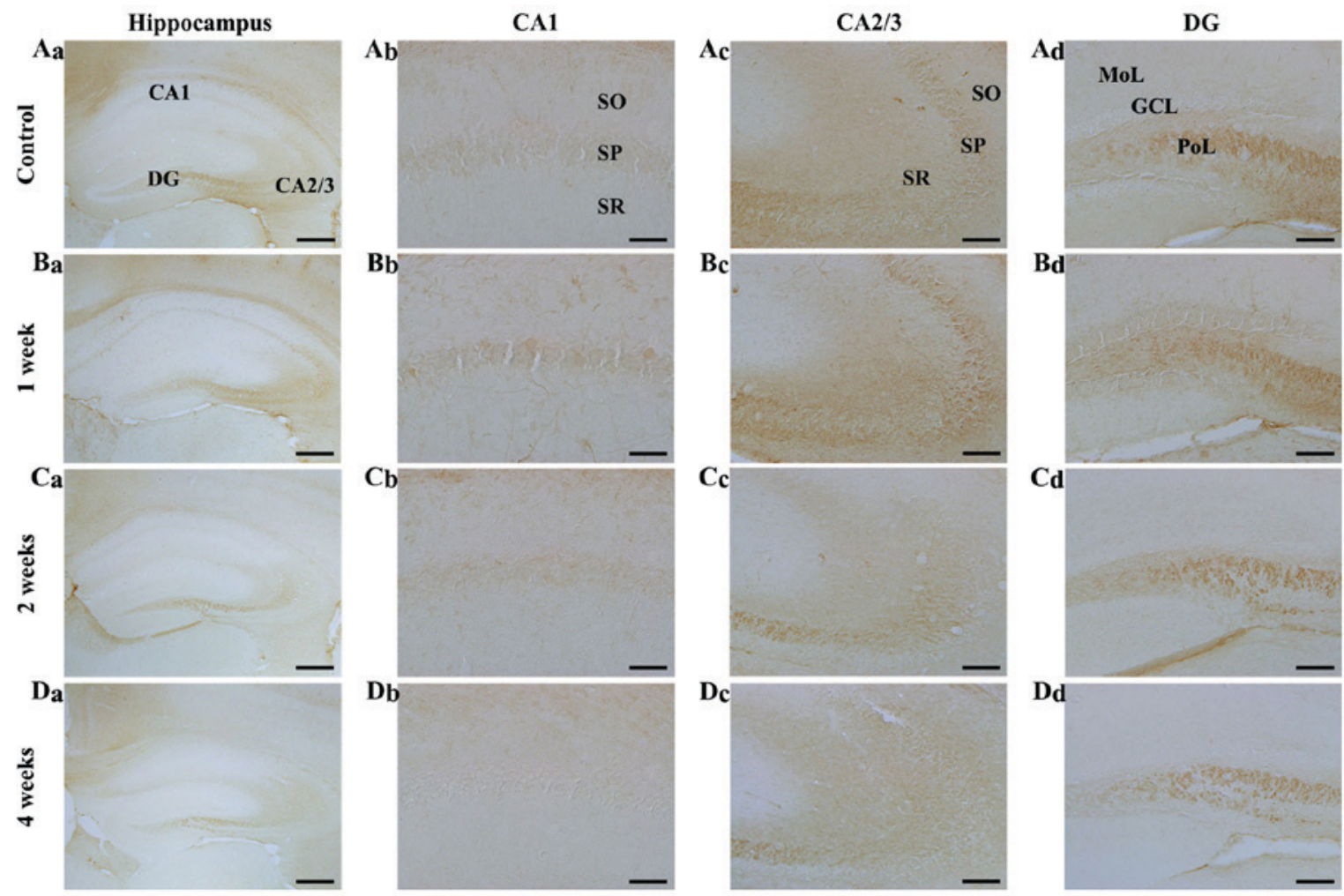

Figure 5. NF-L immunohistochemistry in the hippocampal subregions of the (A) control and SCO-treated mice following (B) 1, (C) 2 and (D) 4 weeks. NF-L immunoreactivity was increased in the (Bc) SP 1 week following SCO treatment; however, NF-L immunoreactivity decreased in all hippocampal subregions, (C and D) 2 and 4 weeks following SCO treatment. Scale bar=800 $\mu \mathrm{m}$ (Aa-Da), $50 \mu \mathrm{m}$ (Ab-Db) and $100 \mu \mathrm{m}$ (Ac-Dc and Ad-Dd). CA, cornus ammonis; DG, dentate gyrus; GCL, granule cell layer; Mol, molecular layer; PoL, polymorphic layer; SO, stratum oriens; SP, stratum pyramidale; SR, stratum radiatum; NF-L, neurofilament-68 kDa; SCO, scopolamine.

avoidance test is a measurement of cognitive memory based on avoidance of a fear-inducing context. Rodents naturally prefer dark compartments; however, receiving an electric shock in a dark compartment causes a conflict with this tendency (37). Furthermore, the Morris water maze test is designed to evaluate spatial learning ability and long-term spatial memory $(38,39)$. We found that chronic systemic SCO treatment reduced the step-through latency in the passive avoidance test, and caused the mice to take a longer time to find the platform than the control group in the Morris water-maze task. Therefore, these findings indicate that chronic systemic treatment with SCO can induce short-term memory deficits, as well as spatial learning and memory impairment.

NFs are key intermediate filaments in neurons and major components of the axonal cytoskeleton $(27,40)$. Alterations of NFs have been observed in neurodegenerative diseases (25-27,41), and disruption of their expression shows important consequences in neuronal function $(26,42)$. In addition, early alterations of NFs represent the development of diffuse axonal injury, and precedes microtubule fracture and depolymerization (43).

A long time ago, Leifer and Kowall reported that selective cellular vulnerability in human cerebral ischemia was related to the reduction of hippocampal pyramidal cells via staining the pyramidal cells with SMI-32, a marker for nonphosphorylated NFs (44). Posmantur et al (45) reported that progressive alterations of NF-L immunoreactivity occurred in dendrites at 15 min after a traumatic brain injury and that a definite loss of dendritic NFs was found 3 to $24 \mathrm{~h}$ after the injury.
Moreover, it has been reported that NFs are disrupted in the hippocampus under depressive states, and the disruption is associated with a dysfunction of hippocampal plasticity in depressive states $(46,47)$. These papers show that alterations in NFs may serve as a trigger for further secondary damage to axons, representing a key insight into a temporal aspect of cytoskeletal degeneration (43).

In the present study, we examined chronological changes in NFs in the mouse hippocampus after chronic systemic treatment with SCO, using the western blot analysis and immunohistochemistry for NFs; we found that NF-H immunoreactivity and levels were significantly increased 1 week after SCO treatment. Thereafter, they significantly decreased with time; the NF-M immunoreactivity and levels gradually decreased and were significantly decreased 4 weeks after the SCO treatment; and NF-L immunoreactivity and levels were significantly decreased from 2 weeks after the SCO treatment. Furthermore, alteration in each immunoreactivity was distinctively different according to the hippocampal subregions and their layers as well as according to the time after SCO treatment. Based on our and the above-mentioned findings, it is obvious that dysfunction in axonal transport might underlie alterations of NFs.

In brief, our present results show that long-term treatment with SCO induced cognitive deficits from 1 week after SCO treatment, although NFs levels and immunoreactivities were significantly and diversely altered in the mouse hippocampus according to the hippocampal subregions and the time-course after the SCO treatment. Therefore, more research needs to be 
conducted about what occurs in cognitive deficits according to an alteration in each NF expression in the hippocampus following chronic systemic treatment with SCO.

\section{Acknowledgements}

The authors would like to thank Mr. Seung Uk Lee for his technical help in this study. This study was supported by Bio-Synergy Research Project (NRF-2015M3A9C4076322) of the Ministry of Science, ICT and Future Planning through the National Research Foundation, by the Bio and Medical Technology Development Program of the NRF funded by the Korean government, MSIP (NRF-2015M3A9B6066835), and by a Priority Research Centers Program grant (NRF-2009-0093812) through the National Research Foundation of Korea funded by the Ministry of Science, ICT and Future Planning.

\section{References}

1. Bartus RT, Dean RL III, Beer B and Lippa AS: The cholinergic hypothesis of geriatric memory dysfunction. Science 217 408-414, 1982

2. Zhou MM, Xue Y, Sun SH, Wen M, Li ZJ, Xu J, Wang JF, Yanagita T, Wang YM and Xue CH: Effects of different fatty acids composition of phosphatidylcholine on brain function of dementia mice induced by scopolamine. Lipids Health Dis 15: 135, 2016.

3. Lee B, Sur B, Shim J, Hahm DH and Lee H: Acupuncture stimulation improves scopolamine-induced cognitive impairment via activation of cholinergic system and regulation of BDNF and CREB expressions in rats. BMC Complement Altern Med 14: $338,2014$.

4. Park JH, Choi HY, Cho JH, Kim IH, Lee TK, Lee JC, Won MH, Chen BH, Shin BN, Ahn JH, et al: Effects of chronic scopolamine treatment on cognitive impairments and myelin basic protein expression in the mouse hippocampus. J Mol Neurosci 59: 579-589, 2016

5. Yoo DY, Choi JH, Kim W, Nam SM, Jung HY, Kim JH, Won MH, Yoon YS and Hwang IK: Effects of luteolin on spatial memory, cell proliferation and neuroblast differentiation in the hippocampal dentate gyrus in a scopolamine-induced amnesia model. Neurol Res 35: 813-820, 2013.

6. Ahmed T and Gilani AH: Inhibitory effect of curcuminoids on acetylcholinesterase activity and attenuation of scopolamine-induced amnesia may explain medicinal use of turmeric in Alzheimer's disease. Pharmacol Biochem Behav 91: 554-559, 2009.

7. Klinkenberg I and Blokland A: The validity of scopolamine as a pharmacological model for cognitive impairment: A review of animal behavioral studies. Neurosci Biobehav Rev 34: $1307-1350,2010$

8. Lian W, Fang J, Xu L, Zhou W, Kang, Xiong W, Jia H, Liu AL and Du GH: DL0410 ameliorates memory and cognitive impairments induced by scopolamine via increasing cholinergic neurotransmission in mice. Molecules 22: pii: E410, 2017.

9. Hami J, Kheradmand $\mathrm{H}$ and Haghir H: Sex differences and laterality of insulin receptor distribution in developing rat hippocampus: An immunohistochemical study. J Mol Neurosci 54: $100-108,2014$

10. Huang W, Cao J, Liu X, Meng F, Li M, Chen B and Zhang J: AMPK plays a dual role in regulation of CREB/BDNF pathway in mouse primary hippocampal cells. J Mol Neurosci 56 : 782-788, 2015

11. Kong Y, Bai PS, Sun H and Nan KJ: Expression of the newly identified gene $\mathrm{CAC} 1$ in the hippocampus of Alzheimer's disease patients. J Mol Neurosci 47: 207-218, 2012.

12. Zhao L, Sun C, Xiong L, Yang Y, Gao Y, Wang L, Zuo H, Xu X, Dong J, Zhou H and Peng R: MicroRNAs: Novel mechanism involved in the pathogenesis of microwave exposure on rats hippocampus. J Mol Neurosci 53: 222-230, 2014.

13. Terry AV Jr, Callahan PM, Hall B and Webster SJ: Alzheimer's disease and age-related memory decline (preclinical). Pharmacol Biochem Behav 99: 190-210, 2011.
14. Gold PE: Acetylcholine modulation of neural systems involved in learning and memory. Neurobiol Learn Mem 80: 194-210, 2003.

15. Hasselmo ME: The role of acetylcholine in learning and memory. Curr Opin Neurobiol 16: 710-715, 2006.

16. Lippa AS, Critchett DJ, Ehlert F, Yamamura HI, Enna SJ and Bartus RT: Age-related alterations in neurotransmitter receptors: An electrophysiological and biochemical analysis. Neurobiol Aging 2: 3-8, 1981

17. Vijayan VK: Cholinergic enzymes in the cerebellum and the hippocampus of the senescent mouse. Exp Gerontol 12: 7-11, 1977.

18. Nixon RA and Sihag RK: Neurofilament phosphorylation: A new look at regulation and function. Trends Neurosci 14: 501-506, 1991.

19. Serrano-Pozo A, Frosch MP, Masliah E and Hyman BT: Neuropathological alterations in Alzheimer disease. Cold Spring Harb Perspect Med 1: a006189, 2011.

20. Dong DL, Xu ZS, Chevrier MR, Cotter RJ, Cleveland DW and Hart GW: Glycosylation of mammalian neurofilaments. Localization of multiple O-linked $\mathrm{N}$-acetylglucosamine moieties on neurofilament polypeptides L and M. J Biol Chem 268: $16679-16687,1993$.

21. Sánchez I, Hassinger L, Sihag RK, Cleveland DW, Mohan P and Nixon RA: Local control of neurofilament accumulation during radial growth of myelinating axons in vivo. Selective role of site-specific phosphorylation. J Cell Biol 151: 1013-1024, 2000.

22. Yabe JT, Chan WK, Chylinski TM, Lee S, Pimenta AF and Shea TB: The predominant form in which neurofilament subunits undergo axonal transport varies during axonal initiation, elongation, and maturation. Cell Motil Cytoskeleton 48: 61-83, 2001.

23. Hayes RL, Yang K, Whitson JS and Postmantur R: Cytoskeletal derangements following central nervous system injury: Modulation by neurotrophic gene transfection. J Neurotrauma 12: 933-941, 1995.

24. Tu PH, Elder G, Lazzarini RA, Nelson D, Trojanowski JQ and Lee VM: Overexpression of the human NFM subunit in transgenic mice modifies the level of endogenous NFL and the phosphorylation state of NFH subunits. J Cell Biol 129: 1629-1640, 1995.

25. Al-Chalabi A and Miller CC: Neurofilaments and neurological disease. Bioessays 25: 346-355, 2003.

26. Julien JP: Neurofilament functions in health and disease. Curr Opin Neurobiol 9: 554-560, 1999.

27. Perrot R, Berges R, Bocquet A and Eyer J: Review of the multiple aspects of neurofilament functions, and their possible contribution to neurodegeneration. Mol Neurobiol 38: 27-65, 2008.

28. Vickers JC, Kirkcaldie MT, Phipps A and King AE: Alterations in neurofilaments and the transformation of the cytoskeleton in axons may provide insight into the aberrant neuronal changes of Alzheimer's disease. Brain Res Bull 126: 324-333, 2016.

29. Siedler DG, Chuah MI, Kirkcaldie MT, Vickers JC and King AE: Diffuse axonal injury in brain trauma: Insights from alterations in neurofilaments. Front Cell Neurosci 8: 429, 2014.

30. Wang X, Wang ZH, Wu YY, Tang H, Tan L, Wang X, Gao XY, Xiong YS, Liu D, Wang JZ and Zhu LQ: Melatonin attenuates scopolamine-induced memory/synaptic disorder by rescuing EPACs/miR-124/Egrl pathway. Mol Neurobiol 47: 373-381, 2013.

31. Yan BC, Park JH, Chen BH, Cho JH, Kim IH, Ahn JH, Lee JC, Hwang IK, Cho JH, Lee YL, et al: Long-term administration of scopolamine interferes with nerve cell proliferation, differentiation and migration in adult mouse hippocampal dentate gyrus, but it does not induce cell death. Neural Regen Res 9: 1731-1739, 2014.

32. Lee JC, Park JH, Ahn JH, Kim IH, Cho JH, Choi JH, Yoo KY, Lee $\mathrm{CH}$, Hwang IK, Cho JH, et al: NNew GABAergic neurogenesis in the hippocampal CA1 region of a gerbil model of long-term survival after transient cerebral ischemic injury. Brain Pathol 26: 581-592, 2016.

33. Park JH, Lee CH, Yoo KY, Choi JH, Hwang IK, Lee JY, Kang IJ and Won MH: FoxO3a immunoreactivity is markedly decreased in the dentate gyrus, not the hippocampus proper, of the aged gerbil. Exp Gerontol 46: 836-840, 2011.

34. Blokland A: Scopolamine-induced deficits in cognitive performance: A review of animal studies. Scopolamine Rev 1: 1-76, 2005.

35. Ebert U and Kirch W: Scopolamine model of dementia: Electroencephalogram findings and cognitive performance. Eur J Clin Invest 28: 944-949, 1998. 
36. Shi Z, Chen L, Li S, Chen S, Sun X, Sun L, Li Y, Zeng J, He Y and Liu X: Chronic scopolamine-injection-induced cognitive deficit on reward-directed instrumental learning in rat is associated with CREB signaling activity in the cerebral cortex and dorsal hippocampus. Psychopharmacology (Berl) 230: 245-260, 2013

37. Romanski LM and LeDoux JE: Information cascade from primary auditory cortex to the amygdala: Corticocortical and corticoamygdaloid projections of temporal cortex in the rat. Cereb Cortex 3: 515-532, 1993.

38. D'Hooge R and De Deyn PP: Applications of the Morris water maze in the study of learning and memory. Brain Res Brain Res Rev 36: 60-90, 2001.

39. Morris R: Developments of a water-maze procedure for studying spatial learning in the rat. J Neurosci Methods 11: 47-60, 1984

40. Fletcher DA and Mullins RD: Cell mechanics and the cytoskeleton. Nature 463: 485-492, 2010.

41. Norgren N, Rosengren L and Stigbrand T: Elevated neurofilament levels in neurological diseases. Brain Res 987: 25-31, 2003.

42. Gotow T: Neurofilaments in health and disease. Med Electron Microsc 33: 173-199, 2000

43. Fournier AJ, Rajbhandari L, Shrestha S, Venkatesan A and Ramesh KT: In vitro and in situ visualization of cytoskeletal deformation under load: traumatic axonal injury. FASEB J 28: $5277-5287,2014$.
44. Leifer D and Kowall NW: Immunohistochemical patterns of selective cellular vulnerability in human cerebral ischemia. J Neurol Sci 119: 217-228, 1993.

45. Posmantur RM, Newcomb JK, Kampfl A and Hayes RL: Light and confocal microscopic studies of evolutionary changes in neurofilament proteins following cortical impact injury in the rat. Exp Neurol 161: 15-26, 2000.

46. Reinés A, Cereseto M, Ferrero A, Bonavita C and Wikinski S: Neuronal cytoskeletal alterations in an experimental model of depression. Neuroscience 129: 529-538, 2004.

47. Sanna MD, Ghelardini $C$ and Galeotti N: Effect of amitriptyline treatment on neurofilament-H protein in an experimental model of depression. Brain Res Bull 128: 1-6, 2017.

(c) (9) This work is licensed under a Creative Commons Attribution 4.0 International (CC BY-NC 4.0) License 